\title{
Ultra-fast and calibration-free temperature sensing in the intrapulse mode
}

\author{
Robin S.M. Chrystie, ${ }^{1, *}$ Ehson F. Nasir, ${ }^{1}$ and Aamir Farooq ${ }^{1}$ \\ ${ }^{l}$ Clean Combustion Research Center, Division of Physical Sciences and Engineering, \\ King Abdullah University of Science and Technology (KAUST), Thuwal, 23955-6900, Saudi Arabia \\ *Corresponding author: robin.chrystie@cantab.net
}

Received Month X, XXXX; revised Month X, XXXX; accepted Month X,

XXXX; posted Month X, XXXX (Doc. ID XXXXX); published Month X, XXXX

\begin{abstract}
A simultaneously time-resolved and calibration-free sensor has been demonstrated to measure temperature at the nanosecond timescale at repetition rates of $1.0 \mathrm{MHz}$. The sensor benefits from relying on a single laser, is intuitive and straightforward to implement, and sweeps across spectral ranges in excess of $1 \mathrm{~cm}^{-1}$. The sensor can fully resolve rovibrational features of the $\mathrm{CO}$ molecule, native to combustion environments, in the mid-infrared range near $\lambda=4.85 \mu \mathrm{m}$ at typical combustion temperatures $(800-2500 \mathrm{~K})$ and pressures $(1-3 \mathrm{~atm})$. All of this is possible through the exploitation of chirp in a quantum cascade laser, operating at a duty cycle of $50 \%$, and by using high bandwidth $(500 \mathrm{MHz})$ photodetection. Here, we showcase uncluttered, spectrally-pure Voigt profile fitting with accompanying peak SNRs of 150, resulting in a typical temperature precision of $0.9 \%(1 \sigma)$ at an effective time-resolution of $1.0 \mathrm{MHz}$. Our sensor is applicable to other species, and can be integrated into commercial technologies. $\quad$ (C) 2013 Optical Society of America

OCIS codes: (320.1590) Ultrafast optics: chirping, (140.0140) Lasers and laser optics: Semiconductor lasers, quantum cascade, (320.1590) Ultrafast optics: nanosecond phenomena, (120.1740) Instrumentation, measurement, and metrology: Combustion diagnostics, (280.3420) Remote sensing and sensors: Laser sensors, (300.6340) Spectroscopy: infrared.
\end{abstract}

Accurate, precise and non-invasive sensing obtainable by laser diagnostics is invaluable to applications such as chemistry, environmental monitoring and combustion research [1-4]. Time-resolved diagnostics are essential to follow transient phenomena, for example, as seen in explosions, internal combustion engines, gas turbines and shock tubes. Here, we focus on the measurement of temperature using a laser diagnostic, in particular the ability to monitor temperature in a highly time-resolved manner. A typical approach involves fixing the injection current [5], and hence wavelength, of two cw semiconductor lasers and directly monitoring, using photodetectors, the absorption of a target species such as $\mathrm{CO}, \mathrm{H}_{2} \mathrm{O}$ or $\mathrm{C}_{2} \mathrm{H}_{4}$ in the gas phase. Such an approach is indeed highly time-resolved, limited only by the speed of the detection system. However, this fixed-wavelength strategy suffers from two problems: uncertainties from (i) drift and jitter of the laser, and (ii) evolution of pressure, emission and composition of the system under measurement. Additionally, the need to use two lasers complicates the optical arrangement and data analysis.

Instead of fixing the wavelength of a cw laser to an absorption peak, wavelength scanning can be invoked to obtain a calibration-free measurement of temperature from a full spectral scan [6]. Greater spectral information reduces uncertainty, since the effects of pressure, emission, composition, and laser instability can be fully accounted for. Conventional rapid-scanned direct absorption relying on sinusoidal or sawtooth modulation can achieve this goal, albeit at relatively low scanning rates (few $\mathrm{kHz}$ ) if adequate spectral coverage of $\sim 1 \mathrm{~cm}^{-1}$ is to be accomplished. The use of intrapulse spectroscopy can combine the advantages of fixed-wavelength and conventional rapid-scanned approaches, simultaneously delivering full spectral measurements $\left(1-3 \mathrm{~cm}^{-1}\right)$ at very high repetition rates $(\sim \mathrm{MHz})$ [7-13].

This Letter examines the ability to achieve highly timeresolved temperature measurements, which are calibration-free, by exploiting the intrapulse mode of excitation. We showcase precise and accurate real-time measurements at pulse repetition rates as high as 1.0 $\mathrm{MHz}$, exceeding that of a preliminary attempt [2] (at 250 $\mathrm{kHz}$ ) in which other hindrances were present. These comprised excessive spectral congestion of rovibrational lines around $1312 \mathrm{~cm}^{-1}$ for $\mathrm{H}_{2} \mathrm{O}$, spectral distortion due to less than ideal effective detection bandwidth (100 MHz), and SNR being limited to a value of only 20 .

Hitherto, the intrapulse mode has not been exploited to render concurrently fast and spectrally-resolved calibration-free measurements of temperature. Provided high bandwidth detection is available, a quasiinstantaneous spectrum can be generated via chirp within a laser pulse. Chirp results from a transient response of applying a step change in injection current to a quantum cascade laser (QCL), for instance. Fig. 1 highlights an example transmittance spectrum (blue trace) from a train of pulses (inset) recorded at a pulse repetition rate of 1.0 $\mathrm{MHz}$ and $50 \%$ duty cycle. Rapid frequency downchirp is exhibited by the étalon trace (FSR: $493 \mathrm{MHz}$ ) in Fig. 1, manifesting a spectral range of $\sim 1 \mathrm{~cm}^{-1}$ generated in 500 ns. The orange trace shows the distribution of localized rates of chirp through the pulse, with a corresponding initial chirp rate of $J_{0}=150 \mathrm{MHz} / \mathrm{ns}$ at the rising edge of the pulse.

The two troughs embedded within the pulse shown in the blue trace of Fig. 1 is due to absorption of $0.5 \% \mathrm{CO}$ in a bath gas of $96.5 \%$ argon and $3 \% \mathrm{H}_{2}$ measured near $\lambda=$ $4.85 \mu \mathrm{m}\left(2060 \mathrm{~cm}^{-1}\right)$ at $1259 \mathrm{~K}$ and $1.18 \mathrm{bar}$, over a path length of $14.2 \mathrm{~cm}$. CO was selected as an absorbing 
species, due to its relatively favorable spectral line separation $\left(0.42 \mathrm{~cm}^{-1}\right)$ and its abundance in combustion applications, rendering it a practical spectral tracer. The elegance of the intrapulse approach here is the ability, in principle, to measure temperature using only a single laser, in a similar vein to our single-detector approach for measuring temperature in Chrystie et al. (2009) [3], whilst also achieving simultaneous calibration-free ultrafast temperature measurements at $\mathrm{MHz}$ rates for the first time.

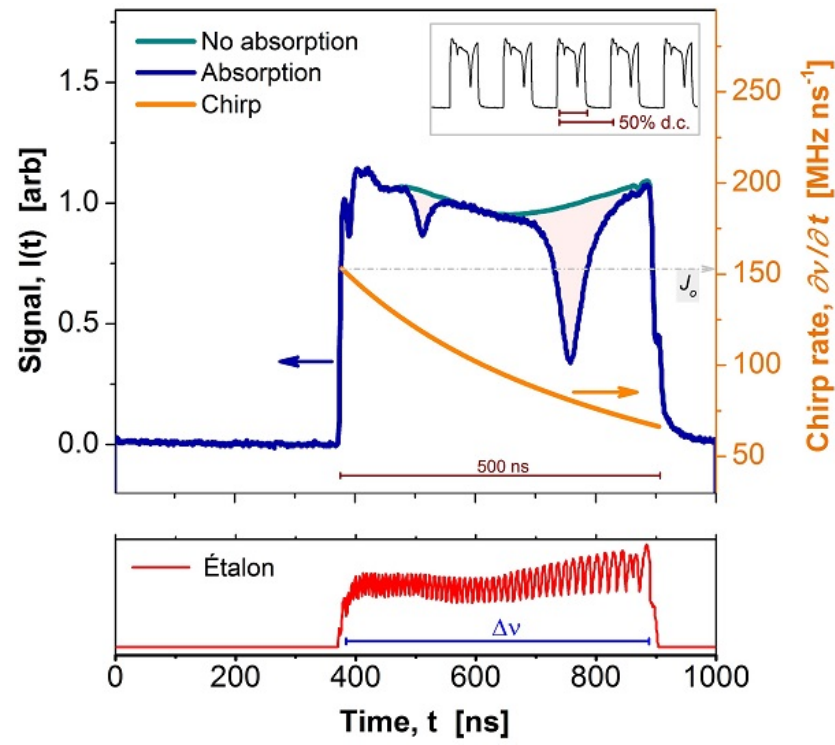

Fig. 1. (Color online) Pulse structure from two CO lines near $4.85 \mu \mathrm{m}$. Conditions of gas: $1259 \mathrm{~K}, 1.18$ bar. Inset shows train of pulses, from which the single pulse is sampled. Etalon pattern corresponds to downchirp of $0.75 \mathrm{~cm}^{-1}$, from where rate of chirp is inferred (orange curve).

Our setup comprised a pulsed DFB QCL (Alpes sb5640), whose nominal wavelength can be tuned by setting laser head temperature and source voltage; such parameters were regulated using controllers (Alpes TC-3) and (GW Instek GPS-3030D), respectively. The pulse timing (i.e., pulse length and duty cycle) was regulated via a function generator (Lecroy WS2052). The laser beam emerging from the head was split using a ZnSe plate, with the secondary reflected beam directed through a solid Germanium étalon towards a high bandwidth $(500 \mathrm{MHz})$ thermoelectrically-cooled $\mathrm{HgCdTe}$ photovoltaic detector (Vigo PVI 4TE-10.6-1×1-TO8- $\mathrm{BaF}_{2}$ ). The primary beam from the ZnSe plate was directed through a shock tube via ZnSe windows towards another similar fast detector. The path traversed through the shock tube corresponds to a diameter of $14.2 \mathrm{~cm}$. The transmitted irradiance through the shock tube is recorded on an oscilloscope (Tektronix DPO4104B, 5GS/s, $1 \mathrm{GHz}$ ). Details of how such a shock tube was operated, in order to generate hot gases, can be found here [2].

Fig. 2 shows an absorbance spectrum, derived using the Beer-Lambert Law, based on a spectrally resolved instantaneous pulse, as exemplified in Fig. 1. The relative frequency scale in $\mathrm{GHz}$ is calibrated from the time axis of the pulse record, as shown in Fig. 1, and the étalonic fringes. The experimental data trace in Fig. 2 (red) spans $0.75 \mathrm{~cm}^{-1}$; enough to cover two rovibrational lines of $\mathrm{CO}$,
$\mathrm{P}(20)$ and $\mathrm{P}(14)$ from the (1-0) and (2-1) bands respectively, near $2060 \mathrm{~cm}^{-1}$ at $1.89 \mathrm{bar}$ and $2377 \mathrm{~K}$. This spectrum is fitted with two Voigt profiles; green and yellow for the low and high energy lines, respectively. Such a large difference in lower-state energies $(\Delta \mathrm{E}$ ' $=$ $1737 \mathrm{~cm}^{-1}$ ) helps to render ensuing temperature measurements more precise, owing to enhanced sensitivity (hence stronger immunity to spectral noise) of relative areas, $\mathrm{R}$, under each transition to changes in temperature, T; viz. $(\mathrm{dR} / \mathrm{R}) /(\mathrm{dT} / \mathrm{T}) \propto \Delta \mathrm{E} " / \mathrm{T}$, derived from differentiating the two-line temperature equation in [2].

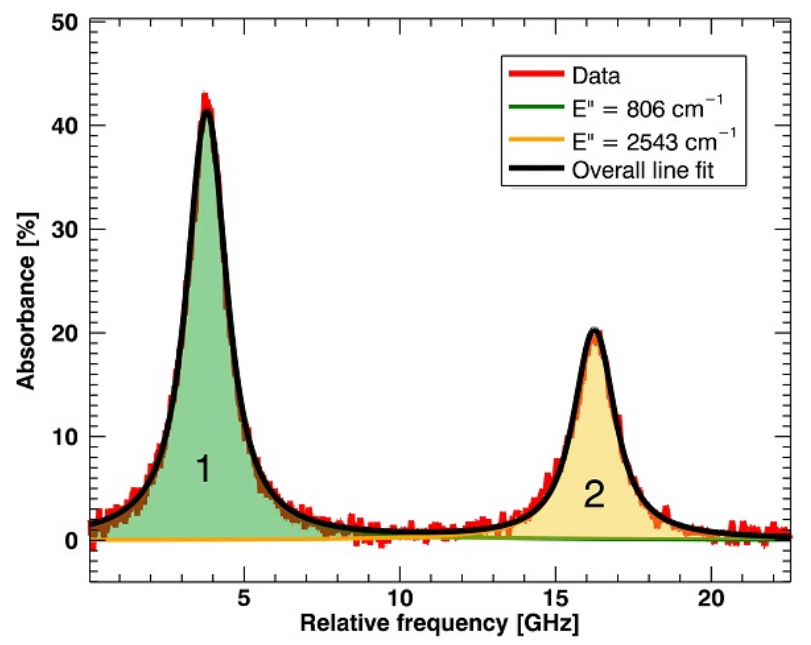

Fig. 2. (Color online) Instantaneous absorbance spectrum (red) derived from a single pulse near $2060 \mathrm{~cm}^{-1}$, spanning $0.75 \mathrm{~cm}^{-1}$. Green and yellow Voigt profiles are fitted to the data, corresponding to gas conditions of $0.5 \% \mathrm{CO}$ in $96.5 \%$ argon and $3 \% \mathrm{H}_{2}, 2377 \mathrm{~K}, 1.89$ bar.

Two lines are identified to be contributing to the spectrum, confirmed by HITRAN [14]. Using HITRAN parameters, and by accounting for spectral resolution, an overall spectral fit can be computed; depicted as the black trace in Fig. 2. Here, spectral resolution (SR) is calculated from the Fourier Transform limit relation [9], related to the chirp rate via: $\mathrm{SR}=\beta(\partial v / \partial \mathrm{t})^{1 / 2}$, where the constant $\beta=$ 0.027 for our system. The spectral resolution is Gaussian, and was thus summed in quadrature to the Dopplertemperature width to correct the absorption profiles. The overall line fit is derived from the summation of the underlying Voigt profiles. Each profile can be simulated by floating the peak and Lorentzian width of the absorption transitions. The summation of these lines results in a fit that is computed from the LevenbergMarquardt algorithm.

Once the fit is complete, a calibration-free measure of the instantaneous temperature can be calculated by integrating Voigt profiles 1 and 2 . The ratio, $R$, of these two areas is used directly in the denominator of the twoline equation [2], in order to calculate calibration-free temperature, T. From inspection of Fig. 2, we observe that a precise spectral fit to the experimental data is possible, even at very high pulse repetition rates of $1.0 \mathrm{MHz}$. It demonstrates that a full spectrum can be generated through transient chirp at the nanosecond timescale, which is essentially instantaneous for many physical processes (e.g. combustion). Despite the speed of the 
sensor demonstrated, there is a surprisingly low level of noise in the spectrum, with a peak SNR of 150 in Fig. 2; this is approximately an order of magnitude improvement over previous measurements [2]. Fig. 2 shows two wellspaced lines, with little overlap between them, and since the Voigt profiles are matching the data well, we can conclude that at these chirp rates and pulse repetition rates, systematic spectral distortion does not pose a problem that would otherwise be caused by insufficient detection bandwidth, as was postulated in our previous study [2].

Hitherto, the datasets discussed have implicitly been based on a $50 \%$ duty cycle, and it is imperative to examine whether this is an ideal setting. Towards properly characterizing the intrapulse method, we investigate how duty cycle affects the amount of downchirp, $\Delta v$. Fig. 3 shows curves of downchirp for particular pulse repetition frequencies as a function of duty cycle. These curves are semi-analytical and the black experimentally-determined datapoints in Fig. 3 match the curves well, suggesting the underlying relationship is valid.

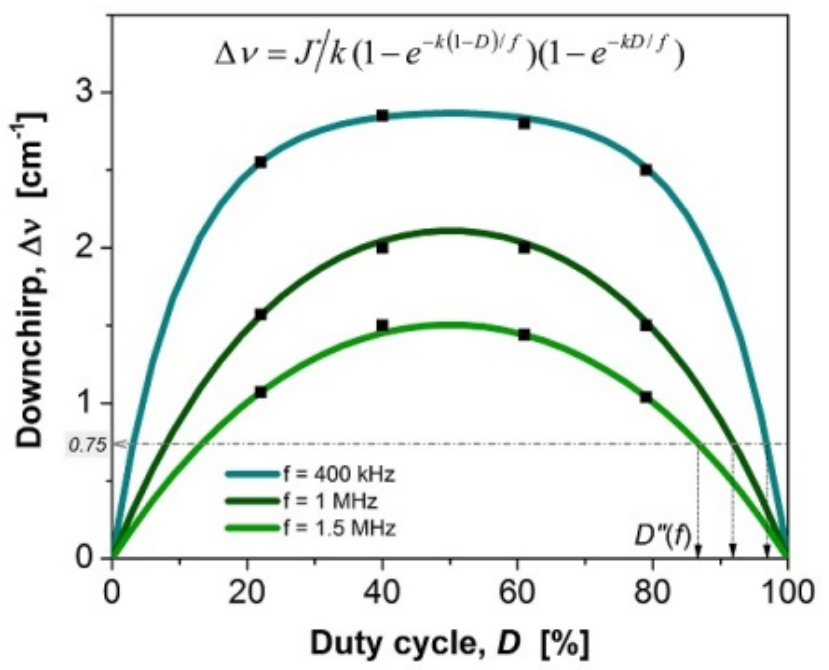

Fig. 3. (Color online) Downchirp as a function of duty cycle, for a source voltage of $11.5 \mathrm{~V}$. $\mathrm{J}^{*}$ and $\mathrm{k}$ refer to the initial chirp rate and exponential decay constant respectively. Each point represents an experimental value. For a downchirp of $0.75 \mathrm{~cm}^{-1}$, there exists two possible sets of $\mathrm{D}$ values; the solution at larger duty cycles, D"(f), is used to seek the optimal spectral resolution in Fig. 4.

The semi-analytical relation is given by Eq. (1), where D is duty cycle, $\mathrm{f}$ is pulse repetition frequency, $\mathrm{k}$ is an exponential decay constant, and $\mathrm{J}^{*}$ is the notional maximum initial chirp rate attainable when the period of time that the pulse is inactive tends toward infinity (i.e. $\mathrm{J}_{\mathrm{o}}$ $\rightarrow \mathrm{J}^{*}$ as $\left.(1-\mathrm{D}) / \mathrm{f} \rightarrow \infty\right)$. $\mathrm{J}^{*}$ only depends on voltage (V), and for our laser was empirically determined to be: $\mathrm{J}^{*}=$ 93V-737.

$$
=J^{*} / k\left(1-e^{-k(1-D) / f}\right)\left(1-e^{-k D / f}\right)
$$

Eq. (1) is derived from integrating the chirp rate (see curve in Fig. 1) across the pulse. It was found experimentally that chirp rate exponentially decays with time after the rising edge of the pulse via a constant decay rate, $\mathrm{k}$. This observation, shown by the orange curve in Fig. 1, is allied with the fact that the chip within the QCL is expected to respond in such a manner to a step change in injection current. It was also found that the assumption of $\mathrm{k}$ being constant held well for pulse durations, $\mathrm{D} / \mathrm{f}<1$ $\mu \mathrm{s}$. The dependency of chirp rate, and hence initial rate $\mathrm{J}_{\mathrm{o}}$, is reflected in Eq. (1). The initial chirp rate, $\mathrm{J}_{0}$, as denoted in Fig. 1, was discovered not to be a simple constant. In fact, $J_{0}$ depends on the history of previous pulses such that the duration for which the injection current is switched-off immediately before the next pulse seems to act as a recovery period for determining the degree of $\mathrm{J}_{\mathrm{o}}$. Our characterization of the laser enabled the dependency of $\mathrm{J}_{0}$ to be determined: $J_{0}=J^{*}\left(1-e^{-k(1-D) / f}\right)$, which forms part of Eq. (1).

We learn from Fig. 3 that the relationship between downchirp and duty cycle is symmetric, such that a $50 \%$ duty cycle delivers maximum downchirp at a given pulse repetition frequency. Predictably, for a fixed voltage V (i.e. fixed $\left.\mathrm{J}^{*}\right), \Delta v$ decreases with a rise in $\mathrm{f}$. We therefore learn that in order to generate the maximum amount of downchirp through the pulse, we need to set the duty cycle to $50 \%$. We have also derived a useful semianalytical relationship (Eq. (1)) to practically predict the amount of spectral range achievable in order to conduct a successful spectroscopic measurement. Each parameter in Eq. (1) can be measured for any semiconductor laser with relative ease.

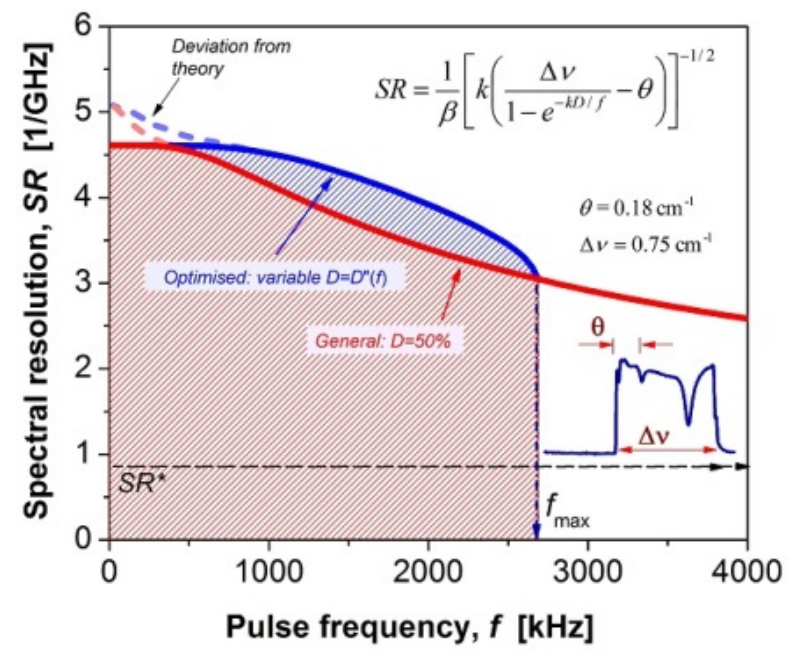

Fig. 4. (Color online) Relationship of spectral resolution with pulse frequency at position $\theta=0.18 \mathrm{~cm}^{-1}$ in the pulse. Red trace corresponds to duty of $50 \%$; blue trace to voltage of $11.5 \mathrm{~V}$ showing optimum SR. Dashed lines show experimental values for $\mathrm{D} / \mathrm{f}>1 \mu \mathrm{s}$. Shaded regions demarcate pulse frequencies below which Beer's Law is applicable.

We have shown $50 \%$ duty cycle to be ideal, yet it is also paramount to check the spectral resolution of our sensor, especially when operating at fast speeds. The red curve in Fig. 4 shows a tradeoff between spectral resolution, SR, and pulse repetition rate, $f$, at a 50\% duty cycle. Specifically, the curve shows the worst-case scenario of lowest SR in our measurements at a spectral location of $\theta$ $=0.18 \mathrm{~cm}^{-1}$ from the rising edge for a pulse width of $\Delta v=$ 
$0.75 \mathrm{~cm}^{-1}$. The blue curve shows an alternative relationship in which voltage (i.e., peak injection current) is fixed at $11.5 \mathrm{~V}$ (maximum voltage that our laser can accept), and duty cycle, $\mathrm{D}$, is a function of $\mathrm{f}$ (i.e. $\mathrm{D}=\mathrm{D}$ "( $\mathrm{f})$ ). It is observed in Fig. 3 for $\Delta v=0.75 \mathrm{~cm}^{-1}$ there exists an upper solution, D"(f) illustrated by the intersection of the ordinate with the three curves; clearly D" increases when $\mathrm{f}$ is reduced.

The red and blue curves originate from the relation for spectral resolution given by Eq. (2), derived from the Fourier Transform limit relation [9]. Eq. (2) predicts SR at a specific spectral position $\theta$ within the pulse. This relationship holds well for $\mathrm{D} / \mathrm{f}<1 \mu \mathrm{s}$, however, the dashed lines in Fig. 4 show measured SR at lower frequencies, $\mathrm{f}$, where pulse duration is too long for $\mathrm{k}$ to remain a true exponential decay constant.

$$
S R=\frac{1}{\beta}\left[k\left(\frac{\Delta v}{1-e^{-k D / f}}-\theta\right)\right]^{-1 / 2}
$$

We learn from Fig. 4 that if the laser is set at its maximum voltage $(11.5 \mathrm{~V})$, the blue curve intersects the red curve (50\% duty cycle) giving the maximum operating frequency of $\mathrm{f}_{\max }=2.7 \mathrm{MHz}$ for this laser at the stated conditions in Fig. 4. Furthermore, Fig. 4 evinces that for all $\mathrm{f}<\mathrm{f}_{\max }$, and across the entire pulse, SR exceeds a critical value of $\mathrm{SR}^{*}\left(0.020 \mathrm{~cm}^{-1}\right)$. The lowest spectral resolution occurs at $\mathrm{f}_{\max }$ with SR giving $0.010 \mathrm{~cm}^{-1}$, which is similar to a high-end FTIR. Critical spectral resolution $\left(\mathrm{SR}^{*}\right)$ is calculated based on a $0.5 \% \mathrm{CO}$ mixture in argon over the spectrally steep flank of the high-energy line at position $\theta$ in the pulse, set with a tolerance of $1 \%$ deviation from the Beer-Lambert Law.

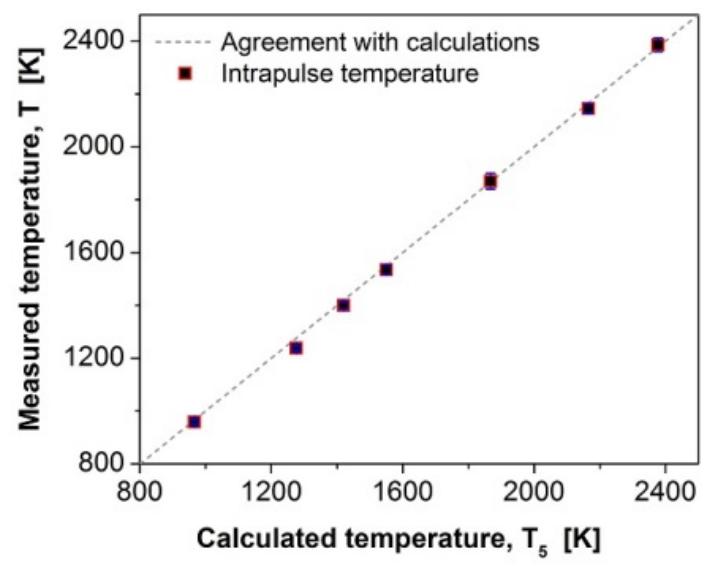

Fig. 5. (Color online) Temperature measured with our method and compared to that from reflected shock waves, $T_{5}$. Each point is an average of 100 instantaneous measurements. Reflected shock pressures, $\mathrm{P}_{5}$, ranged $0.8-2.5$ atm.

Given a thorough characterization based on duty cycle and spectral resolution, as a final endeavor to confirm our temperature sensor's ability to measure at very high speeds $(\sim 1.0 \mathrm{MHz})$, we show Fig. 5 . Here, there is a direct comparison of measured temperature, $\mathrm{T}$, versus temperature, $\mathrm{T}_{5}$, calculated from standard 1-D shock relations. Each black datapoint represents an average of 100 instantaneous spectra (at $1 \mathrm{MHz}$ rate). Error bars are shown, but are not prominent due to their small size. There is excellent agreement over the 966 - $2377 \mathrm{~K}$ range with a typical absolute deviation of $\sim 0.7 \%$ from $\mathrm{T}_{5}$, and a typical precision of $0.9 \%(1 \sigma)$ (no binning: effective rate of $1.0 \mathrm{MHz}$ ).

In conclusion, we demonstrated the ability to easily resolve rovibrational features at pressures of $\sim 2 \mathrm{~atm}$ and at high temperatures relevant to combustion. We can measure temperature instantaneously using a single laser, over a nanosecond timescale repetitively at rates of $1.0 \mathrm{MHz}$. Our sensor exhibited relatively little noise using high bandwidth detection, with SNRs exceeding 100. We concluded that the ideal duty cycle for practical operation in the intrapulse mode is $50 \%$. It was also shown that at relatively high pulse repetition rates, and hence larger chirp rates, the spectral resolution through the pulse is more than sufficient to ensure that the Beer-Lambert Law remains accurate. Finally, we confirm the validity of the measurements from our sensor through agreements with well-known temperatures behind reflected shock waves. This sensor is an example of how the intrapulse approach is gaining increasing recognition of its utility in applied research, notwithstanding potential commercial application.

The authors would like to thank Dr. Olivier Landry from Alpes Lasers for advice. The authors would like to acknowledge King Abdullah University of Science and Technology (KAUST) and Saudi Basic Industries Corporation (SABIC) for research funds towards this project.

\section{References}

1. M.G. Allen, Meas. Sci. Technol. 9, 545 (1998).

2. R.S.M. Chrystie, E.F. Nasir, and A. Farooq, Proc. Combust. Inst. 10.1016/j.proci.2014.06.069 (2014).

3. R.S.M. Chrystie, I.S. Burns, J. Hult, and C.F. Kaminski, Opt. Lett. 34, 2492 (2009).

4. R.S.M. Chrystie, I.S. Burns, and C.F. Kaminski, Combust. Sci. Technol. 185, 180 (2013).

5. H. Li, A. Farooq, J.B. Jeffries, and R.K. Hanson, Appl. Phys. B. 89, 407 (2007).

6. G. Wysocki, M. McCurdy, S. So, D. Weidmann, C. Roller, R.F. Curl, F.K. Tittel, Appl. Opt. 43, 6040 (2004).

7. E. Normand, M. McCulloch, G. Duxbury, and N. Langford, Opt. Lett. 28, 16 (2003).

8. A. Hangauer, G. Spinner, M. Nikodem, and G. Wysocki, Appl. Phys. Lett. 103, 191107 (2013).

9. J.H. van Helden, S.J. Horrocks, and G.A.D. Ritchie, Appl. Phys. Lett. 92, 081506 (2008).

10. J.H. Northern, G.A.D. Ritchie, E.P. Smakman, J.H. van Helden, J. Cockburn, and G. Duxbury, Opt. Lett. 35, 2750 (2010).

11. J.A. Nwaboh, O. Werhahn, and D. Schiel, Appl. Phys. B. 103, 947 (2011).

12. J. Manne, W. Jager, and J. Tulip, Appl. Phys. B. 94, 337 (2009).

13. B. Grouiez, V. Zeninari, L. Joly, and B. Parvitte, Appl. Phys. B 100, 265 (2010).

14. C. Richard, I.E. Gordon, L.S. Rothman, M. Abel, L. Frommhold, M. Gustafsson, J.M. Hartmann, C. Hermans, W.J. Lafferty, G.S. Orton, K.M. Smith, and H. Tran, J. Quant. Spectrosc. Radiat. Transfer 113, 1276 (2012). 
1. M.G. Allen, "Diode laser absorption sensors for gas-dynamic and combustion flows" Meas. Sci. Technol. 9, 545 (1998).

2. R.S.M. Chrystie, E.F. Nasir, and A. Farooq, "Towards simultaneous calibration-free and ultra-fast sensing of temperature and species in the intrapulse mode" Proc. Combust. Inst. 10.1016/j.proci.2014.06.069 (2014).

3. R.S.M. Chrystie, I.S. Burns, J. Hult, and C.F. Kaminski, "High-repetition-rate combustion thermometry with two-line atomic fluorescence excited by diode lasers" Opt. Lett. 34, 2492 (2009).

4. R.S.M. Chrystie, I.S. Burns, and C.F. Kaminski, "Temperature response of an acoustically-forced turbulent lean premixed flame: A quantitative experimental determination" Combust. Sci. Technol. 185, 180 (2013).

5. H. Li, A. Farooq, J.B. Jeffries, and R.K. Hanson, "Near-infrared diode laser absorption sensor for rapid measurements of temperature and water vapor in a shock tube” Appl. Phys.. B. 89, 407 (2007).

6. G. Wysocki, M. McCurdy, S. So, D. Weidmann, C. Roller, R.F. Curl, F.K. Tittel, "Pulsed quantumcascade laser-based sensor for trace-gas detection of carbonyl sulfide" Appl. Opt. 43, 6040 (2004).

7. E. Normand, M. McCulloch, G. Duxbury, and N. Langford, "Fast, real-time spectrometer based on a pulsed quantum-cascade laser" Opt. Lett. 28, 16 (2003).

8. A. Hangauer, G. Spinner, M. Nikodem, and G. Wysocki, "Chirped Laser Dispersion Spectroscopy with Directly Modulated Quantum Cascade Laser" Appl. Phys. Lett. 103, 191107 (2013).

9. J.H. van Helden, S.J. Horrocks, and G.A.D. Ritchie, "Application of quantum cascade lasers in studies of low-pressure plasmas: Characterization of rapid passage effects on density and temperature measurements" Appl. Phys. Lett. 92, 081506 (2008).

10. J.H. Northern, G.A.D. Ritchie, E.P. Smakman, J.H. van Helden, J. Cockburn, and G. Duxbury, "Rapid passage signals induced by chirped quantum cascade laser radiation: $K$ state dependent-delay effects in the v2 band of NH3" Opt. Lett. 35, 2750 (2010).

11. J.A. Nwaboh, O. Werhahn, and D. Schiel, "Measurement of $\mathrm{CO}$ amount fractions using a pulsed quantum-cascade laser operated in the intrapulse mode” Appl. Phys. B. 103, 947 (2011).

12. J. Manne, W. Jager, and J. Tulip, "Sensitive detection of ammonia and ethylene with a pulsed quantum cascade laser using intra- and inter- pulse spectroscopic techniques" Appl. Phys. B. 94, $337 \quad$ (2009).

13. B. Grouiez, V. Zeninari, L. Joly, and B. Parvitte, "Pulsed quantum cascade laser spectroscopy with intermediate-size pulses: application to NH3 in the $10 \mu \mathrm{m}$ region” Appl. Phys. B 100, 265 (2010).

14. C. Richard, I.E. Gordon, L.S. Rothman, M. Abel, L. Frommhold, M. Gustafsson, J.M. Hartmann, C. Hermans, W.J. Lafferty, G.S. Orton, K.M. Smith, and H. Tran, J. Quant. Spectrosc. Radiat. Transfer 113, 1276 (2012). 